\title{
Equilibria and steering laws for planar formations ${ }^{\text {t3 }}$
}

\author{
E.W. Justh ${ }^{\mathrm{a}, *}$, P.S. Krishnaprasad ${ }^{\mathrm{a}, \mathrm{b}}$ \\ ${ }^{a}$ Institute for Systems Research, University of Maryland, College Park, MD 20742, USA \\ ${ }^{\mathrm{b}}$ Department of Electrical and Computer Engineering, University of Maryland, College Park, MD 20742, USA
}

Received 4 September 2003; received in revised form 12 October 2003; accepted 26 October 2003

\begin{abstract}
This paper presents a Lie group setting for the problem of control of formations, as a natural outcome of the analysis of a planar two-vehicle formation control law. The vehicle trajectories are described using the planar Frenet-Serret equations of motion, which capture the evolution of both the vehicle position and orientation for unit-speed motion subject to curvature (steering) control. The set of all possible (relative) equilibria for arbitrary $G$-invariant curvature controls is described (where $G=S E(2)$ is a symmetry group for the control law), and a global convergence result for the two-vehicle control law is proved. An $n$-vehicle generalization of the two-vehicle control law is also presented, and the corresponding (relative) equilibria for the $n$-vehicle problem are characterized. Work is on-going to discover stability and convergence results for the $n$-vehicle problem. (c) 2003 Elsevier B.V. All rights reserved.
\end{abstract}

Keywords: Moving frames; Formations; Relative equilibria; Stability

\section{Introduction}

This work is motivated by the problem of multiple vehicle formation (or swarm) control, e.g., for meter-scale unmanned aerial vehicles (UAVs) [7]. The objective is to use automatic control to avoid collisions between vehicles, maintain cohesiveness of the formation, be robust to loss of individuals, and scale favorably to large swarms. Models of the individual

\footnotetext{
This research was supported in part by the Naval Research Laboratory under Grants N00173-02-1G002 and N00173-03-1G001, by the Air Force Office of Scientific Research under AFOSR Grant No. F49620-01-0415, and by the Army Research Office under ODDR\&E MURI01 Program Grant No. DAAD19-01-1-0465 to the Center for Communicating Networked Control Systems (through Boston University).

* Corresponding author.

E-mail addresses: justh@isr.umd.edu (E.W. Justh), krishna@isr.umd.edu (P.S. Krishnaprasad).
}

vehicles are based on the planar Frenet-Serret equations of motion, which describe how vehicle trajectories evolve under curvature (steering) control, subject to a unit-speed assumption [1]. While our interest is primarily in formation control for small UAVs, this approach to modeling and control could also be applicable to formations or swarms of ground vehicles or underwater vehicles, and may also be a useful starting point for understanding certain features of biological swarming or schooling behavior.

This paper presents a Lie group setting for the problem of control of formations. The setting emerges naturally from the analysis of basic cases and concomitant physical constraints on the controls. (A modern reference for control systems on Lie groups is Jurdjevic [6].)

The primary analytical result we present (in Section 3 ) is a global convergence result for a planar two-vehicle formation control law, proved using 
LaSalle's Invariance Principle [9]. A related convergence result for a single vehicle responding to a fixed beacon is also presented in Section 4. A generalization of the two-vehicle control law to $n$ vehicles is presented in Section 5, but a stability analysis is the subject of on-going work, and will be presented in a future paper.

The two-vehicle control law has an evident Lie group structure, and this structure is exploited (in Section 3 ) to determine the set of all possible (relative) equilibria for arbitrary $G$-invariant curvature controls, where $G=S E(2)$ is the symmetry group for the control law. Ideas of "shape control" on Lie groups arise in a natural way for this problem $[10,12,20]$. The equilibrium analysis is also generalized to the $n$-vehicle problem (in Section 5). Finally, in Section 6, we discuss directions for future research.

The use of curvature controls can be given the mechanical interpretation of steering unit-mass, unit-charge particles by magnetic fields (and hence by forces derived from vector potentials). For a discussion of the general theory of such controls, see [19]. This is in contrast with other current approaches to formation control that are based on scalar potentials, both in the point particle [14] and rigid-body [16] setting. Furthermore, our emphasis on the Lie group structure of the control laws distinguishes our work from an established physics literature in the area of large collections of interacting particles subject to local interaction laws, and giving rise to pattern-forming systems, spatially localized coherent structures (e.g., flocks), and phase transitions (e.g., from disorder to order); see, for instance, $[3,11,17,18]$.

\section{Modeling planar formations}

The motion of a charged particle in a magnetic field is determined by the Lorentz force law,

$m \ddot{\mathbf{r}}=\frac{q}{c}(\dot{\mathbf{r}} \times B)$,

where $\mathbf{r} \in \mathbb{R}^{3}$ is the position of the particle, $m$ is its mass, $q$ is its charge, $B$ is the magnetic field, and $c$ is the speed of light. The corresponding Lagrangian is

$L=\frac{1}{2} m|\dot{\mathbf{r}}|^{2}+\frac{q}{c}(\dot{\mathbf{r}} \cdot A)$,

where the vector potential $A=A(\mathbf{r})$ satisfies $B=\nabla \times$ $A$. The force exerted on the charged particle by the magnetic field is gyroscopic: the kinetic energy of the particle, $H_{\mathrm{L}}=\frac{1}{2} m|\dot{\mathbf{r}}|^{2}$, is conserved. If the particle motion is restricted to the $x-y$ plane, and the magnetic field $B=\left(00 B_{z}\right)^{\mathrm{T}}$ is perpendicular to the plane of motion, then Eq. (1) may be written as

$\ddot{\mathbf{r}}=\left[\begin{array}{cc}0 & -u \\ u & 0\end{array}\right] \dot{\mathbf{r}}$,

where $u=-q B_{z} / m c$ and $\mathbf{r} \in \mathbb{R}^{2}$. On the level set of $H_{\mathrm{L}}$ given by $H_{\mathrm{L}}=m / 2$, the particle moves at unit speed, and Eq. (3) is equivalent to the planar Frenet-Serret equations of motion,

$\dot{\mathbf{r}}=\mathbf{x}$,

$\dot{\mathbf{x}}=\mathbf{y} u$,

$\dot{\mathbf{y}}=-\mathbf{x} u$,

where $\mathbf{x}$ is the unit tangent vector to the particle trajectory, $\mathbf{y}$ is the unit normal vector, and $u$ is the curvature (or steering) control.

Eq. (4) describes the evolution of both the position $\mathbf{r}$ of the particle and its orientation, given by the orthonormal frame (x,y). Eq. (4) can thus be thought of as a dynamical system evolving on $S E(2)$, the group of rigid motions in the plane. Formations of vehicles are then treated as interacting particles in $S E(2)$ : each particle obeys a copy of Eq. (4), with its steering control $u$ determined by an interaction law. The problem is to determine interaction laws which achieve formations.

For the UAV application, we are naturally led to consider constant-speed motion, because air speed must be maintained for such vehicles to remain aloft. Furthermore, maneuverability is limited for such vehicles, and turning involves significant energy expenditure (sideslip while banking must be overcome with additional thrust). Therefore, formation control laws for UAVs should in some sense (at least approximately) minimize the curvature of the vehicle trajectories. We are thus led to consider three critical performance issues: (1) formation cohesiveness; (2) collision avoidance; and (3) steering "energy" minimization (e.g., for a single vehicle, the steering "energy" could be defined as the $L^{2}$-norm of the steering control).

It is interesting to note that similar practical considerations apply to certain ground vehicles (and 
water-surface vehicles), as well. Maintaining high speeds can provide dynamic stability, which is reduced while steering, and thus it is reasonable to penalize steering "energy". Our modeling framework based on the planar Frenet-Serret equations is a natural one in which to address the issue of steering energy minimization, because the curvatures of the trajectories are precisely the control signals. In this paper, we present control laws which directly address the issues of formation cohesiveness and collision avoidance. The freedom remaining (e.g., through choice of parameters) in the control laws could be used to address the issue of steering energy minimization, and this is a topic of on-going work.

\section{A two-vehicle formation control law}

Fig. 1(a) illustrates the trajectories of two vehicles, and their respective planar Frenet-Serret frames. Consider the coupled dynamics of the vehicles given by

$$
\begin{aligned}
& \dot{\mathbf{r}}_{1}=\mathbf{x}_{1}, \quad \dot{\mathbf{r}}_{2}=\mathbf{x}_{2}, \\
& \dot{\mathbf{x}}_{1}=\mathbf{y}_{1} u_{1}, \quad \dot{\mathbf{x}}_{2}=\mathbf{y}_{2} u_{2}, \\
& \dot{\mathbf{y}}_{1}=-\mathbf{x}_{1} u_{1}, \quad \dot{\mathbf{y}}_{2}=-\mathbf{x}_{2} u_{2}, \\
& u_{1}=-\eta(|\mathbf{r}|)\left(-\frac{\mathbf{r}}{|\mathbf{r}|} \cdot \mathbf{x}_{1}\right)\left(-\frac{\mathbf{r}}{|\mathbf{r}|} \cdot \mathbf{y}_{1}\right) \\
& \quad-f(|\mathbf{r}|)\left(-\frac{\mathbf{r}}{|\mathbf{r}|} \cdot \mathbf{y}_{1}\right)+\mu(|\mathbf{r}|) \mathbf{x}_{2} \cdot \mathbf{y}_{1},
\end{aligned}
$$
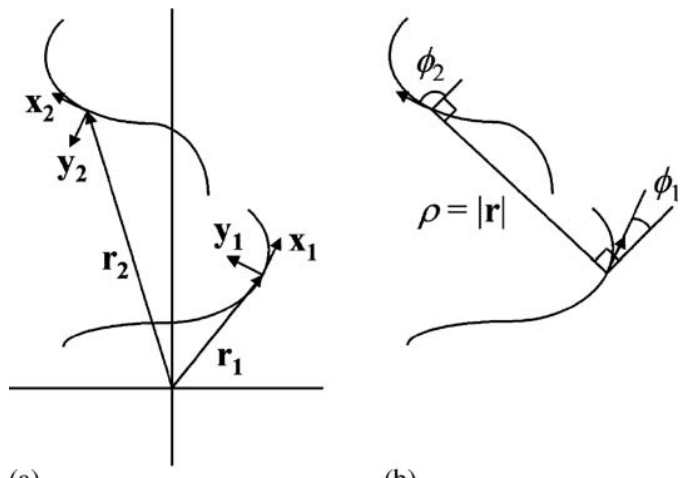

(a)

(b)

Fig. 1. Planar trajectories for two vehicles and their respective planar Frenet-Serret frames: (a) in $\left(\mathbf{r}_{1}, \mathbf{x}_{1}, \mathbf{y}_{1}\right),\left(\mathbf{r}_{2}, \mathbf{x}_{2}, \mathbf{y}_{2}\right)$ coordinates; and (b) in $\left(\rho, \phi_{1}, \phi_{2}\right)$ coordinates, as defined in Section 3.1 .

$$
\begin{aligned}
u_{2}= & -\eta(|\mathbf{r}|)\left(\frac{\mathbf{r}}{|\mathbf{r}|} \cdot \mathbf{x}_{2}\right)\left(\frac{\mathbf{r}}{|\mathbf{r}|} \cdot \mathbf{y}_{2}\right) \\
& -f(|\mathbf{r}|)\left(\frac{\mathbf{r}}{|\mathbf{r}|} \cdot \mathbf{y}_{2}\right)+\mu(|\mathbf{r}|) \mathbf{x}_{1} \cdot \mathbf{y}_{2},
\end{aligned}
$$

where $\mathbf{r}=\mathbf{r}_{2}-\mathbf{r}_{1}$, the functions $\eta(\cdot), \mu(\cdot)$, and $f(\cdot)$ are Lipschitz continuous, and $f(\cdot)$ satisfies

$$
\begin{aligned}
& \lim _{\rho \rightarrow 0} f(\rho)=-\infty, \\
& \lim _{\rho \rightarrow \infty} \int_{\tilde{\rho}}^{\rho} f(\hat{\rho}) \mathrm{d} \hat{\rho}=\infty, \quad \text { for some } \tilde{\rho}>0 .
\end{aligned}
$$

We further assume that $\mu(|\mathbf{r}|)>\frac{1}{2} \eta(|\mathbf{r}|)>0$ for all $|\mathbf{r}| \geqslant 0$.

Although the form of Eq. (5) may appear complicated, it is actually simple to interpret, straightforward to implement, and has both a discrete (relabling) symmetry, and an $S E(2)$ symmetry, which have both practical and theoretical importance. To simplify the explanation, suppose that

$f(|\mathbf{r}|)=\alpha\left[1-\left(\frac{r_{0}}{|\mathbf{r}|}\right)^{2}\right]$

and $\alpha, \eta, \mu$, and $r_{0}$ are all positive constants. In the expressions for the steering controls $u_{1}$ and $u_{2}$, the terms involving $\mu$ serve to align the heading directions of the vehicles. The terms involving $f(|\mathbf{r}|)$ serve to steer the vehicles away from one another if they are too close (i.e., if $|\mathbf{r}|<r_{0}$ ), or to steer them toward each other if they are too far apart (i.e., if $|\mathbf{r}|>r_{0}$ ). The terms involving $\eta$ serve to align each vehicle perpendicular to the baseline between the two vehicles. Note that the control law given by Eq. (5) is invariant under vehicle relabling. This coincides with the intuitive notion that both vehicles "run the same algorithm".

\subsection{Shape variables}

Identifying (punctured) $\mathbb{R}^{2}$ with the (punctured) complex plane, we define

$$
\begin{aligned}
& \mathbf{r}=\mathbf{r}_{2}-\mathbf{r}_{1}=|\mathbf{r}| \mathrm{ie}^{\mathrm{i} \psi}, \\
& \mathbf{x}_{1}=\mathrm{e}^{\mathrm{i} \theta_{1}}, \quad \mathbf{x}_{2}=\mathrm{e}^{\mathrm{i} \theta_{2},} \\
& \phi_{1}=\theta_{1}-\psi, \quad \phi_{2}=\theta_{2}-\psi,
\end{aligned}
$$


where we note that $\psi+\pi / 2$ is the argument (i.e., angle) of $\mathbf{r}$. We introduce the variable

$\rho=|\mathbf{r}|$,

and we observe that

$\frac{\mathbf{r}}{|\mathbf{r}|} \cdot \mathbf{x}_{1}=\operatorname{Re}\left\{\mathrm{ie}^{\mathrm{i} \psi}\left(\mathrm{e}^{\mathrm{i} \theta_{1}}\right)^{*}\right\}=\sin \phi_{1}$,

$\frac{\mathbf{r}}{|\mathbf{r}|} \cdot \mathbf{x}_{2}=\sin \phi_{2}$,

$\frac{\mathbf{r}}{|\mathbf{r}|} \cdot \mathbf{y}_{1}=\operatorname{Re}\left\{\mathrm{ie}^{\mathrm{i} \psi}\left(\mathrm{ie}^{\mathrm{i} \theta_{1}}\right)^{*}\right\}=\cos \phi_{1}$,

$\frac{\mathbf{r}}{|\mathbf{r}|} \cdot \mathbf{y}_{2}=\cos \phi_{2}$.

Fig. 1(b) illustrates the definitions of $\rho, \phi_{1}$, and $\phi_{2}$.

Using

$\dot{\mathbf{r}}=\mathrm{ie}^{\mathrm{i} \psi} \frac{\mathrm{d}}{\mathrm{d} t}|\mathbf{r}|-|\mathbf{r}| \mathrm{e}^{\mathrm{i} \psi} \dot{\psi}=\mathrm{e}^{\mathrm{i} \theta_{2}}-\mathrm{e}^{\mathrm{i} \theta_{1}}$

we find that

$\dot{\rho}=\sin \phi_{2}-\sin \phi_{1}$,

$\dot{\psi}=-\frac{1}{\rho}\left(\cos \phi_{2}-\cos \phi_{1}\right)$.

We also have

$\dot{\theta}_{1}=u_{1}=-\eta(\rho) \sin \phi_{1} \cos \phi_{1}+f(\rho) \cos \phi_{1}$

$$
+\mu(\rho) \sin \left(\theta_{2}-\theta_{1}\right)
$$

$\dot{\theta}_{2}=u_{2}=-\eta(\rho) \sin \phi_{2} \cos \phi_{2}-f(\rho) \cos \phi_{2}$

$$
+\mu(\rho) \sin \left(\theta_{1}-\theta_{2}\right)
$$

We thus obtain the system

$\dot{\rho}=\sin \phi_{2}-\sin \phi_{1}$,

$\dot{\phi}_{1}=-\eta(\rho) \sin \phi_{1} \cos \phi_{1}+f(\rho) \cos \phi_{1}$

$$
+\mu(\rho) \sin \left(\phi_{2}-\phi_{1}\right)+\frac{1}{\rho}\left(\cos \phi_{2}-\cos \phi_{1}\right),
$$

$\dot{\phi}_{2}=-\eta(\rho) \sin \phi_{2} \cos \phi_{2}-f(\rho) \cos \phi_{2}$

$$
\begin{aligned}
& +\mu(\rho) \sin \left(\phi_{1}-\phi_{2}\right) \\
& +\frac{1}{\rho}\left(\cos \phi_{2}-\cos \phi_{1}\right) .
\end{aligned}
$$

System (14) represents a reduction of dynamics (5) by the symmetry group $S E(2)$, which is made possible

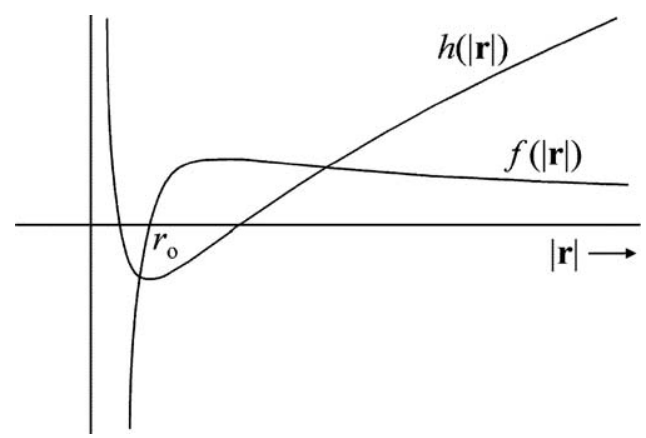

Fig. 2. An example of suitable functions $f(\cdot)$ and $h(\cdot)$, for purposes of Proposition 1.

by the fact that the control law of Eq. (5) depends only on suitably defined shape variables, i.e., it is invariant under an action of the symmetry group $S E(2)$. This reduction has been shown concretely through the use of the shape variables $\rho, \phi_{1}$, and $\phi_{2}$, which depend only on relative positions and orientations. The natural formulation of the formation-control problem in terms of group variables for $S E(2)$, and a precise formulation of the reduction from configuration space to shape space, is deferred to Section 3.3.

\subsection{Convergence result}

Consider the Lyapunov function candidate

$V_{\text {pair }}=-\ln \left(\cos \left(\phi_{2}-\phi_{1}\right)+1\right)+h(\rho)$,

where $f(\rho)=\mathrm{d} h / \mathrm{d} \rho$ (see Fig. 2). Observe that the term $-\ln \left(\cos \left(\phi_{2}-\phi_{1}\right)+1\right)$ penalizes heading-angle differences between the two vehicles, and the term $h(\rho)$ penalizes vehicle separations which are too large or too small. Differentiating $V_{\text {pair }}$ with respect to time along trajectories of (14) gives

$$
\begin{aligned}
\dot{V}_{\text {pair }}= & \frac{\sin \left(\phi_{2}-\phi_{1}\right)}{\cos \left(\phi_{2}-\phi_{1}\right)+1}\left(\dot{\phi}_{2}-\dot{\phi}_{1}\right)+f(\rho) \dot{\rho} \\
= & \frac{\sin \left(\phi_{2}-\phi_{1}\right)}{\cos \left(\phi_{2}-\phi_{1}\right)+1} \\
& \times\left[-\eta(\rho)\left(\sin \phi_{2} \cos \phi_{2}-\sin \phi_{1} \cos \phi_{1}\right)\right. \\
& +2 \mu(\rho) \sin \left(\phi_{1}-\phi_{2}\right)
\end{aligned}
$$




$$
\begin{aligned}
& \left.-f(\rho)\left(\cos \phi_{2}+\cos \phi_{1}\right)\right] \\
& +f(\rho)\left(\sin \phi_{2}-\sin \phi_{1}\right) \\
= & \frac{-\sin \left(\phi_{2}-\phi_{1}\right)}{\cos \left(\phi_{2}-\phi_{1}\right)+1}\left[2 \mu(\rho) \sin \left(\phi_{2}-\phi_{1}\right)\right. \\
& \left.+\frac{\eta(\rho)}{2}\left(\sin 2 \phi_{2}-\sin 2 \phi_{1}\right)\right],
\end{aligned}
$$

where we have used the identity

$$
\begin{aligned}
& \sin \left(\phi_{2}-\phi_{1}\right)\left(\cos \phi_{2}+\cos \phi_{1}\right) \\
& \quad-\left(\cos \left(\phi_{2}-\phi_{1}\right)+1\right)\left(\sin \phi_{2}-\sin \phi_{1}\right)=0 .
\end{aligned}
$$

We also have the identity

$$
\begin{aligned}
\sin \left(\phi_{2}-\phi_{1}\right)\left(\sin \left(\phi_{2}-\phi_{1}\right)+\frac{1}{2}\left(\sin 2 \phi_{2}-\sin 2 \phi_{1}\right)\right) \\
=\frac{1}{2}\left[\left(\cos \phi_{1}+\cos \phi_{2}\right)^{2}\left(\sin \phi_{1}-\sin \phi_{2}\right)^{2}\right. \\
\left.\quad+\left(\sin ^{2} \phi_{1}-\sin ^{2} \phi_{2}\right)^{2}\right] .
\end{aligned}
$$

Therefore, provided $\mu(\rho)>\frac{1}{2} \eta(\rho), \forall \rho \geqslant 0$, it follows that $\dot{V}_{\text {pair }} \leqslant 0$, and $\dot{V}_{\text {pair }}=0$ if and only if $\sin \left(\phi_{2}-\right.$ $\left.\phi_{1}\right)=0$. We can use this calculation to prove a convergence result.

Proposition 1. Consider the system given by Eq. (14), evolving on $\mathbb{R} \times \mathbb{T}^{2}$, where $\mathbb{T}^{2}$ is the two-torus (i.e., $\phi_{i}+2 \pi$ is identified with $\phi_{i}, i=1,2$ ). In addition, assume the following:

1. $\eta(\rho), \mu(\rho)$, and $f(\rho)$ are Lipschitz continuous on $(0, \infty)$

2. $f(\rho)=\mathrm{d} h / \mathrm{d} \rho$, so that $h(\rho)$ is continuously differentiable on $(0, \infty)$;

3. $\lim _{\rho \rightarrow 0} h(\rho)=\infty, \lim _{\rho \rightarrow \infty} h(\rho)=\infty$, and $\exists \tilde{\rho}$ such that $h(\tilde{\rho})=0$;

4. $\eta(\rho)>0, \mu(\rho)>0$, and $\mu(\rho)>\frac{1}{2} \eta(\rho)$, for all $\rho \geqslant 0$.

\section{Define the set}

$\Lambda=\left\{\left(\rho, \phi_{1}, \phi_{2}\right)|| \phi_{1}-\phi_{2} \mid \neq \pi\right.$ and $\left.0<\rho<\infty\right\}$.

Then any trajectory starting in $\Lambda$ converges to the set of equilibrium points for system (14).

Proof. Observe that $V_{\text {pair }}$ given by Eq. (15) is continuously differentiable on $\Lambda$. By assumption (2) and the form of $V_{\text {pair }}$, we conclude that $V_{\text {pair }}$ is radially unbounded (i.e., $V_{\text {pair }} \rightarrow \infty$ as $\left|\phi_{1}-\phi_{2}\right| \rightarrow \pi$, as $\rho \rightarrow 0$, or as $\rho \rightarrow \infty)$. Therefore, for each trajectory starting in $\Lambda$ there exists a compact sublevel set $\Omega$ of $V_{\text {pair }}$ such that the trajectory remains in $\Omega$ for all future time. Then by LaSalle's Invariance Principle [9], the trajectory converges to the largest invariant set $M$ of the set $E$ of all points in $\Omega$ where $\dot{V}_{\text {pair }}=0$. The set $E$ in this case is the set of all points $\left(\rho, \phi_{1}, \phi_{2}\right) \in \Omega$ such that $\sin \left(\phi_{1}-\phi_{2}\right)=0$, i.e., such that $\phi_{1}=\phi_{2}$ (since $\Omega \subset \Lambda$ ). At points in $E$, the dynamics may be expressed as

$\dot{\rho}=0$,

$\dot{\phi}_{1}=-\left[\eta(\rho) \sin \phi_{1}+f(\rho)\right] \cos \phi_{1}$,

$\dot{\phi}_{2}=-\left[\eta(\rho) \sin \phi_{1}-f(\rho)\right] \cos \phi_{1}$.

If $\phi_{1} \neq \pm \pi / 2$ and $f(\rho) \neq 0$, then the trajectory leaves $E$. The largest invariant set contained in $E$ may thus be expressed as

$$
\begin{aligned}
M= & \left(\left\{\left(\rho_{e}, 0,0\right) \mid f\left(\rho_{e}\right)=0\right\}\right. \\
& \cup\left\{\left(\rho_{e}, \pi, \pi\right) \mid f\left(\rho_{e}\right)=0\right\} \\
& \cup\left\{\left(\rho, \frac{\pi}{2}, \frac{\pi}{2}\right), \forall \rho\right\} \\
& \left.\cup\left\{\left(\rho,-\frac{\pi}{2},-\frac{\pi}{2}\right), \forall \rho\right\}\right) \cap \Omega,
\end{aligned}
$$

which is simply the set of equilibria of system (14) contained in $\Omega$.

Remark. While $V_{\text {pair }}$ penalizes the difference in heading angles between the two vehicles, it does not favor any particular orientation of the vehicles relative to the baseline between them. It is thus necessary to examine the maximal invariant set $M$ to determine possible equilibrium shapes of the formation.

Remark. Suppose, e.g., that $f(\cdot)$ is given by Eq. (7), with $\alpha=\eta=\mu=1$, and $r_{0} \geqslant 1$. Then the set of equilibria which system (14) can converge to consists of the isolated points $\left(r_{0}, 0,0\right)$ and $\left(r_{0}, \pi, \pi\right)$, the connected set $(\rho, \pi / 2, \pi / 2)$, and the connected set $(\rho,-\pi / 2,-\pi / 2)$. The isolated points $\left(r_{0}, 0,0\right)$, and $\left(r_{0}, \pi, \pi\right)$ are easily interpreted: both vehicles are heading in the same direction, perpendicular to the baseline between them, and separated by a distance $r_{0}$. These stable equilibria minimize $V_{\text {pair }}$. The other sets of equilibria correspond to the two vehicles heading in the same direction, one 
leading the other by a distance $\rho$ (with the two sets distinguished by which vehicle is in the lead). These equilibria can be shown to be linearly unstable.

\subsection{Lie group formulation}

We have introduced a model for formations based on unit-speed motion with steering control, and we have shown how the types of formation control laws we consider can be interpreted physically as point particles interacting through gyroscopic forces (recall the charged particle and magnetic field analogy). We then presented a specific control law for a pair of vehicles, and gave a physical interpretation of the various terms in the control law. Indeed, the two-vehicle control law embodies characteristics of many biological swarming and schooling models, which tend to have: (1) some mechanism for heading alignment; (2) switching between attraction or repulsion based on separation distance; and (3) greater responsiveness for small separations [4].

One of the key steps of the analysis was the identification of certain shape variables, representing relative angles and relative displacements. By analyzing the shape dynamics, we were able to identify and determine the stability of equilibrium shapes. For two vehicles, the shape variables $\rho, \phi_{1}$, and $\phi_{2}$ are sufficient. However, for more than two vehicles, it is important to use the correct generalization of the idea of shape variables, and this leads naturally to a "shape-control" problem in the Lie group setting $[10,12,20]$. We can view the dynamics of system (5) as evolving on a (collision-free) configuration submanifold $M_{\text {config }} \subset$ $G \times G$, where $G=S E(2)$ is the special Euclidean group in the plane. Specifically, if $g_{1}, g_{2} \in G$ are represented as

$$
g_{1}=\left[\begin{array}{ccc}
\mathbf{x}_{1} & \mathbf{y}_{1} & \mathbf{r}_{1} \\
0 & 0 & 1
\end{array}\right], \quad g_{2}=\left[\begin{array}{lll}
\mathbf{x}_{2} & \mathbf{y}_{2} & \mathbf{r}_{2} \\
0 & 0 & 1
\end{array}\right],
$$

then

$M_{\text {config }}=\left\{\left(g_{1}, g_{2}\right) \in G \times G \mid \mathbf{r}_{1} \neq \mathbf{r}_{2}\right\}$.

(From Proposition 1, we can conclude that for initial conditions in $M_{\text {config }}$, the dynamics evolve in $M_{\text {config }}$ for all future time, provided the controls given by Eq. (5) are used. However, for purposes of this section, we are primarily interested in identifying relative equilibria without restriction to a particular control law.) We can express the dynamics given by Eq. (5) as

$\dot{g}_{1}=g_{1} \xi_{1}=g_{1}\left(A_{0}+A_{1} u_{1}\right)$,

$\dot{g}_{2}=g_{2} \xi_{2}=g_{2}\left(A_{0}+A_{1} u_{2}\right)$,

where $\xi_{1}, \xi_{2} \in \mathfrak{g}=$ the Lie algebra of $G$, and the matrices $A_{0}$ and $A_{1}$ (which generate $\mathfrak{g}$ under Lie bracketing) are given by

$A_{0}=\left[\begin{array}{lll}0 & 0 & 1 \\ 0 & 0 & 0 \\ 0 & 0 & 0\end{array}\right], \quad A_{1}=\left[\begin{array}{ccc}0 & -1 & 0 \\ 1 & 0 & 0 \\ 0 & 0 & 0\end{array}\right]$.

We define

$g=g_{1}^{-1} g_{2}$,

and observe that

$$
\begin{aligned}
g & =\left[\begin{array}{cc}
\mathbf{x}_{1}^{\mathrm{T}} & -\mathbf{r}_{1} \cdot \mathbf{x}_{1} \\
\mathbf{y}_{1}^{\mathrm{T}} & -\mathbf{r}_{1} \cdot \mathbf{y}_{1} \\
0 & 1
\end{array}\right]\left[\begin{array}{ccc}
\mathbf{x}_{2} & \mathbf{y}_{2} & \mathbf{r}_{2} \\
0 & 0 & 1
\end{array}\right] \\
& =\left[\begin{array}{ccc}
\mathbf{x}_{1} \cdot \mathbf{x}_{2} & \mathbf{x}_{1} \cdot \mathbf{y}_{2} & \left(\mathbf{r}_{2}-\mathbf{r}_{1}\right) \cdot \mathbf{x}_{1} \\
\mathbf{y}_{1} \cdot \mathbf{x}_{2} & \mathbf{y}_{1} \cdot \mathbf{y}_{2} & \left(\mathbf{r}_{2}-\mathbf{r}_{1}\right) \cdot \mathbf{y}_{1} \\
0 & 0 & 1
\end{array}\right] .
\end{aligned}
$$

Similarly,

$$
\begin{aligned}
g^{-1} & =g_{2}^{-1} g_{1} \\
& =\left[\begin{array}{ccc}
\mathbf{x}_{1} \cdot \mathbf{x}_{2} & \mathbf{x}_{2} \cdot \mathbf{y}_{1} & -\left(\mathbf{r}_{2}-\mathbf{r}_{1}\right) \cdot \mathbf{x}_{2} \\
\mathbf{y}_{2} \cdot \mathbf{x}_{1} & \mathbf{y}_{1} \cdot \mathbf{y}_{2} & -\left(\mathbf{r}_{2}-\mathbf{r}_{1}\right) \cdot \mathbf{y}_{2} \\
0 & 0 & 1
\end{array}\right] .
\end{aligned}
$$

Let $g_{i j}$ denote the elements of the matrix $g$, let $g^{i j}$ denote the elements of the matrix $g^{-1}$, and let

$r=\sqrt{g_{13}^{2}+g_{23}^{2}}=\sqrt{\left(g^{13}\right)^{2}+\left(g^{23}\right)^{2}}$.

We have the following relationships among the $g_{i j}$ and $g^{i j}$ :

$$
\begin{aligned}
& g_{11}=g_{22}=g^{11}=g^{22}, \\
& g_{12}=-g_{21}=g^{21}=-g^{12}, \\
& g_{11}^{2}+g_{12}^{2}=1 .
\end{aligned}
$$


Remark. We can express $u_{1}$ and $u_{2}$ as defined by Eq. (5) as

$$
\begin{aligned}
u_{1}(g)= & -\eta(r)\left(\frac{g_{13} g_{23}}{r^{2}}\right)+f(r)\left(\frac{g_{23}}{r}\right)+\mu(r) g_{21} \\
u_{2}(g)= & -\eta(r)\left(\frac{g^{13} g^{23}}{r^{2}}\right) \\
& +f(r)\left(\frac{g^{23}}{r}\right)+\mu(r) g^{21} .
\end{aligned}
$$

Eqs. (23), (25), and (30) are sufficient to conclude that the closed-loop dynamics are $G$-invariant. Although we do not require the specific control law given by Eq. (30) for the (relative) equilibrium analysis presented in this subsection, we do assume that the control law is $G$-invariant, in addition to ensuring that the dynamics evolve in the collision-free submanifold $M_{\text {config. }}$.

The dynamics for $g$ are given by

$$
\begin{aligned}
\dot{g} & =-g_{1}^{-1} \dot{g}_{1} g_{1}^{-1} g_{2}+g_{1}^{-1} \dot{g}_{2} \\
& =-g_{1}^{-1} g_{1} \xi_{1} g+g_{1}^{-1} g_{2} \xi_{2} \\
& =-\xi_{1} g+g \xi_{2} \\
& =g\left(\xi_{2}-g^{-1} \xi_{1} g\right) \\
& =g\left(\xi_{2}-\operatorname{Ad}_{g^{-1}} \xi_{1}\right) \\
& =g \xi,
\end{aligned}
$$

where $\xi=\xi_{2}-\operatorname{Ad}_{g^{-1}} \xi_{1} \in \mathfrak{g}$. If the feedback controls depend only on the shape variable $g$, then $\xi=\xi(g)$ and (31) evolves on the shape manifold $M_{\text {shape }}=M_{\text {config }} / G$.

We refer to (31) as the reduced dynamics. Equilibria of the reduced dynamics correspond to relative equilibria of the full dynamics in $M_{\text {config. The }}$ equilibria $g_{e}$ of the reduced dynamics are found by setting

$\xi\left(g_{e}\right)=\xi_{2}\left(g_{e}\right)-g_{e}^{-1} \xi_{1}\left(g_{e}\right) g_{e}=0$.

Eq. (32) is equivalent to

$g_{e} \xi_{2}\left(g_{e}\right)=\xi_{1}\left(g_{e}\right) g_{e}$, which (dropping the " $e$ " subscript) becomes

$$
\begin{aligned}
& {\left[\begin{array}{ccc}
g_{11} & g_{12} & g_{13} \\
-g_{12} & g_{11} & g_{23} \\
0 & 0 & 1
\end{array}\right]\left[\begin{array}{ccc}
0 & -u_{2} & 1 \\
u_{2} & 0 & 0 \\
0 & 0 & 0
\end{array}\right]} \\
& =\left[\begin{array}{ccc}
0 & -u_{1} & 1 \\
u_{1} & 0 & 0 \\
0 & 0 & 0
\end{array}\right]\left[\begin{array}{ccc}
g_{11} & g_{12} & g_{13} \\
-g_{12} & g_{11} & g_{23} \\
0 & 0 & 1
\end{array}\right] .
\end{aligned}
$$

Eq. (34) simplifies to

$$
\begin{aligned}
& {\left[\begin{array}{ccc}
g_{12} u_{2} & -g_{11} u_{2} & g_{11} \\
g_{11} u_{2} & g_{12} u_{2} & -g_{12} \\
0 & 0 & 0
\end{array}\right]} \\
& \quad=\left[\begin{array}{ccc}
g_{12} u_{1} & -g_{11} u_{1} & 1-g_{23} u_{1} \\
g_{11} u_{1} & g_{12} u_{1} & g_{13} u_{1} \\
0 & 0 & 0
\end{array}\right] .
\end{aligned}
$$

Since $g_{11}^{2}+g_{12}^{2}=1$, at equilibrium we must have

$$
u_{2}=u_{1}, \quad g_{11}=1-g_{23} u_{1}, \quad g_{12}=-g_{13} u_{1} .
$$

But Eq. (32) is also equivalent to

$$
\xi_{2}\left(g_{e}\right) g_{e}^{-1}=g_{e}^{-1} \xi_{1}\left(g_{e}\right),
$$

which (by an analogous computation) leads to

$u_{2}=u_{1}, \quad g_{11}=1-g^{23} u_{2}, \quad g_{12}=g^{13} u_{2}$.

Using $g_{11}^{2}+g_{12}^{2}=1$, from (36) we obtain

$$
\begin{aligned}
1 & =\left(1-g_{23} u_{1}\right)^{2}+g_{13}^{2} u_{1}^{2} \\
& =1-2 g_{23} u_{1}+g_{23}^{2} u_{1}^{2}+g_{13}^{2} u_{1}^{2}, \\
0 & =u_{1}\left[\left(g_{13}^{2}+g_{23}^{2}\right) u_{1}-2 g_{23}\right]
\end{aligned}
$$

so that

$u_{1}=\frac{2 g_{23}}{g_{13}^{2}+g_{23}^{2}}, \quad$ or $\quad u_{1}=0$.

There are thus two distinct equilibrium cases to analyze: $u_{1}=0$ and $u_{1}=2 g_{23} / r^{2}$. First, consider the case $u_{1}=0$. Then from Eqs. (36) and (38), we conclude

$$
u_{2}=u_{1}=0, \quad g_{11}=1, \quad g_{12}=0 .
$$

For such an equilibrium, $\mathbf{x}_{1}=\mathbf{x}_{2}$, so we also have

$g_{13}=-g^{13}, \quad g_{23}=-g^{23}$. 
However, the relationship between $\mathbf{x}_{1}$ and $\mathbf{r}=\mathbf{r}_{2}-\mathbf{r}_{1}$ is arbitrary.

Next, consider the case $u_{1}=2 g_{23} / r^{2} \neq 0$. We must assume that $r>0$ so that $u_{1}$ is well-defined. Then from Eqs. (36) and (38), we conclude

$u_{2}=u_{1}=2 g_{23} / r^{2}$,

$g_{23}=g^{23}, \quad g_{13}=-g^{13}$,

$g_{11}=1-2 g_{23}^{2} / r^{2}, \quad g_{12}=-2 g_{13} g_{23} / r^{2}$.

With some further calculation, one can show that the corresponding relative equilibrium consists of the two vehicles moving on the same circular orbit, separated by a chord of fixed length [7].

We summarize the results of the forgoing calculations in the following proposition:

Proposition 2. Consider the dynamics given by Eq. (23), evolving on the collision-free submanifold

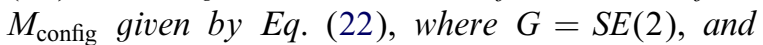
where $\mathbf{r}_{1}$ and $\mathbf{r}_{2}$ are defined by Eq. (21). Assume that the controls $u_{1}$ and $u_{2}$ depend only on the shape variable g given by Eq. (25) (i.e., the controls are G-invariant). Then for equilibrium shapes (i.e., for relative equilibria of the dynamics (23) on configuration space) $u_{1}=u_{2}$, and there are only two possibilities:

(a) $u_{1}=u_{2}=0$ at equilibrium, in which case a relative equilibrium consists of both vehicles heading in the same direction (with arbitrary relative positions), or

(b) $u_{1}=u_{2}=2 g_{23} / r^{2} \neq 0$, in which case a relative equilibrium consists of both vehicles moving on the same circular orbit separated by a chord of fixed length.

Proof. Follows from the calculations above. (For more details, see [7].)

Remark. The notion of shape space associated to a symmetry group is useful for describing the evolution of system $(23)[10,12,20]$. To summarize, Eq. (23) describes motion in the configuration space. The variable $g=g_{1}^{-1} g_{2}$ plays the role of a shape variable, and evolves on the (collision-free) shape submanifold $M_{\text {shape }}=M_{\text {config }} / G$. The reduced dynamics on shape space are given by Eq. (31). Equilibria $g_{e}$ of the reduced dynamics correspond to equilibrium shapes. The configuration variables can be expressed in terms of the shape variable as

$\left(g_{1}, g_{2}\right)=\left(g_{1}, g_{1} g\right)$

and if $g=g_{e}$ (i.e., the shape is an equilibrium shape), then the trajectory in the configuration space is a relative equilibrium.

Remark. The collision-free submanifold $M_{\text {shape }}$ is diffeomorphic to (punctured) $\mathbb{R} \times \mathbb{T}^{2}$, the set which appears in the statement of Proposition 1. Indeed, Proposition 1 can be understood as a convergence result for the shape dynamics.

Remark. The Lyapunov function $V_{\text {pair }}$ given by Eq. (15) is, as expected, also $G$-invariant, and can be expressed as

$$
V_{\text {pair }}=-\ln \left[1-\frac{g_{13} g^{13}+g_{23} g^{23}}{r^{2}}\right]+h(r) .
$$

\section{Control law for one vehicle (and beacon)}

In the previous section, we studied a two-vehicle control law for which the group of all rigid motions in the plane, $S E(2)$, was a symmetry group. We showed that for all initial conditions (except a thin set corresponding to the vehicles heading initially in exactly opposite directions), the system converges to a formation in which both vehicles head off in the same direction, perpendicular to the baseline between them, and separated by a distance prescribed by the function $f(\cdot)$. However, the particular direction that the vehicles head off in as the shape variables converge is determined by initial conditions. In practical situations, it is often desirable to direct the formation toward some particular location, e.g., toward a (fixed) beacon. Introducing a beacon, however, breaks the $S E(2)$ symmetry of the control law.

In this section, we consider a control law for a single vehicle responding to a fixed beacon. The target equilibrium "shape" is for the vehicle to follow a circular trajectory with the beacon at the center. The translational symmetry of $S E(2)$ is broken, but the rotational symmetry remains, and so descending from 


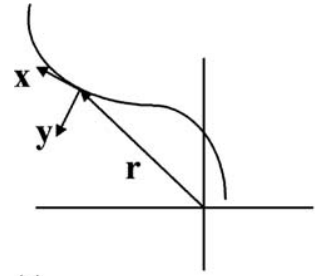

(a)

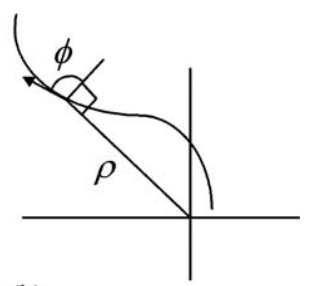

(b)

Fig. 3. Planar trajectory and Frenet-Serret frame for a single vehicle responding to a fixed beacon: (a) in $(\mathbf{r}, \mathbf{x}, \mathbf{y})$ coordinates; and (b) in $(\rho, \phi)$ coordinates.

configuration space to shape space amounts to a reduction by the circle group, $S^{1}$.

\subsection{Shape variables}

The steering control for a single vehicle is based on its position and orientation relative to a "beacon" fixed at the origin, as illustrated in Fig. 3(a).

$\dot{\mathbf{r}}=\mathbf{x}$,

$\dot{\mathbf{x}}=\mathbf{y} u$,

$\dot{\mathbf{y}}=-\mathbf{x} u$,

$u=-\eta(|\mathbf{r}|)\left(\frac{\mathbf{r}}{|\mathbf{r}|} \cdot \mathbf{x}\right)\left(\frac{\mathbf{r}}{|\mathbf{r}|} \cdot \mathbf{y}\right)$

$$
-f(|\mathbf{r}|)\left(\frac{\mathbf{r}}{|\mathbf{r}|} \cdot \mathbf{y}\right) \text {. }
$$

The physical interpretation for system (46) is similar to that of system (5). However, one of the two vehicles is replaced by the beacon, and because there is no notion of "heading direction" associated with the beacon, we set $\mu=0$ to eliminate the heading-alignment term present in (5).

Define

$\mathbf{r}=|\mathbf{r}| \mathrm{ie}^{\mathrm{i} \psi}, \quad \mathbf{x}=\mathrm{e}^{\mathrm{i} \theta}, \quad \rho=|\mathbf{r}|, \quad \phi=\theta-\psi$.

(The variables $\rho$ and $\phi$ are illustrated in Fig. 3(b).) Then we obtain, analogously to system (14),

$\dot{\rho}=\sin \phi$,

$\dot{\phi}=-\eta(\rho) \sin \phi \cos \phi-\left(f(\rho)-\frac{1}{\rho}\right) \cos \phi$.

\subsection{Convergence result}

Consider the Lyapunov function candidate

$V_{\text {single }}=-\ln (\cos \phi)+h(\rho)$,

defined on the set

$\Lambda_{\text {single }}=\left\{(\rho, \phi) \mid \rho>0,-\frac{\pi}{2}<\phi<\frac{\pi}{2}\right\}$

and where $\mathrm{d} h / \mathrm{d} \rho=f(\rho)-1 / \rho$. Observe that the term $-\ln (\cos \phi)$ penalizes deviations in the vehicle heading direction from the direction perpendicular to the baseline between the vehicle and beacon. The term $h(\rho)$ penalizes vehicle-to-beacon distances which are too large or too small.

The derivative of $V_{\text {single }}$ along trajectories of (48) is

$$
\begin{aligned}
\dot{V}_{\text {single }}= & \left(\frac{\sin \phi}{\cos \phi}\right) \dot{\phi}+\left(f(\rho)-\frac{1}{\rho}\right) \dot{\rho} \\
= & \frac{\sin \phi}{\cos \phi}\left[-\eta(\rho) \sin \phi \cos \phi-\left(f(\rho)-\frac{1}{\rho}\right) \cos \phi\right] \\
& +\left(f(\rho)-\frac{1}{\rho}\right) \sin \phi \\
= & -\eta(\rho) \sin ^{2} \phi .
\end{aligned}
$$

Based on this calculation, we can prove a convergence result.

Proposition 3. Consider the system given by Eq. (48), evolving on $\mathbb{R} \times \mathbb{T}^{1}$, where $\mathbb{T}^{1}$ is the one-torus (i.e., $\phi+2 \pi$ is identified with $\phi$ ). In addition, assume the following:

1. $\eta(\rho)$ and $f(\rho)$ are Lipschitz continuous on $(0, \infty)$;

2. $\mathrm{d} h / \mathrm{d} \rho=f(\rho)-1 / \rho$, so that $h(\rho)$ is continuously differentiable on $(0, \infty)$;

3. $\lim _{\rho \rightarrow 0} h(\rho)=\infty, \lim _{\rho \rightarrow \infty} h(\rho)=\infty$ and $\exists \tilde{\rho}$ such that $h(\tilde{\rho})=0$;

4. $\eta(\rho)>0, \forall \rho \geqslant 0$.

Let $\Lambda_{\text {single }}$ be defined by Eq. (50). Then any trajectory starting in $\Lambda_{\text {single }}$ converges to the set of equilibrium points for system (48), i.e., the set

$$
\Gamma_{e}=\left\{(\rho, \phi) \mid\left(f(\rho)-\frac{1}{\rho}\right)=0, \phi=0\right\} .
$$


Proof. Observe that $V_{\text {single }}$ given by Eq. (49) is continuously differentiable on $\Lambda_{\text {single }}$. By assumption (2) and the form of $V_{\text {single }}$, we conclude that $V_{\text {single }}$ is radially unbounded (i.e., $V_{\text {single }} \rightarrow \infty$ as $|\phi| \rightarrow \pi / 2$, as $\rho \rightarrow 0$, or as $\rho \rightarrow \infty)$. Therefore, for each trajectory starting in $\Lambda_{\text {single }}$ there exists a compact sublevel set $\Omega$ of $V_{\text {single }}$ such that the trajectory remains in $\Omega$ for all future time. Then by LaSalle's Invariance Principle [9], the trajectory converges to the largest invariant set $M$ of the set $E$ of all points in $\Omega$ where $\dot{V}_{\text {single }}=0$. The set $E$ in this case is the set of all points $(\rho, \phi) \in \Omega$ such that $\sin (\phi)=0$, i.e., such that $\phi=0$ ( since $\left.\Omega \subset \Lambda_{\text {single }}\right)$. At points in $E$, the dynamics may be expressed as

$\dot{\rho}=0$,

$\dot{\phi}=-\left(f(\rho)-\frac{1}{\rho}\right)$.

If $(f(\rho)-1 / \rho) \neq 0$, then the trajectory leaves $E$. The largest invariant set contained in $E$ may thus be expressed as

$M=\Gamma_{e} \cap \Omega$,

which is simply the set of equilibria of system (48) contained in $\Omega$.

Remark. If the set $\Gamma_{e}$ consists of isolated points, then Proposition 3 implies that each trajectory starting in $\Lambda_{\text {single }}$ converges to an equilibrium point.

Remark. A result analogous to Proposition 3 holds for trajectories starting in

$\Lambda_{\text {single }}^{\text {alt }}=\left\{(\rho, \phi)|\rho>0,| \phi \mid>\frac{\pi}{2}\right\}$.

Instead of $V_{\text {single }}$ defined by Eq. (49), the Lyapunov function

$V_{\text {single }}^{\text {alt }}=-\ln (-\cos \phi)+h(\rho)$

is used.

\section{Control laws for multiple vehicles}

The control system given by Eq. (5) can be generalized to $n$ vehicles as follows:

$\dot{\mathbf{r}}_{j}=\mathbf{x}_{j}$,

$\dot{\mathbf{x}}_{j}=\mathbf{y}_{j} u_{j}$,

$\dot{\mathbf{y}}_{j}=-\mathbf{x}_{j} u_{j}$ with

$$
\begin{aligned}
u_{j}= & \frac{1}{n} \sum_{k \neq j}\left[-\eta\left(\frac{\mathbf{r}_{j k}}{\left|\mathbf{r}_{j k}\right|} \cdot \mathbf{x}_{j}\right)\left(\frac{\mathbf{r}_{j k}}{\left|\mathbf{r}_{j k}\right|} \cdot \mathbf{y}_{j}\right)\right. \\
& \left.-f\left(\left|\mathbf{r}_{j k}\right|\right)\left(\frac{\mathbf{r}_{j k}}{\left|\mathbf{r}_{j k}\right|} \cdot \mathbf{y}_{j}\right)+\mu \mathbf{x}_{k} \cdot \mathbf{y}_{j}\right],
\end{aligned}
$$

$j=1, \ldots, n$, where $\mathbf{r}_{j k}=\mathbf{r}_{j}-\mathbf{r}_{k}$. Observe that each vehicle trajectory evolves with its own planar FrenetSerret equation, and that the control is simply an average of terms analogous to those present in system (5).

A generalization of the energy function $V_{\text {pair }}$ that might play a role in analyzing the convergence of this system is

$V_{n}=\sum_{j=1}^{n} \sum_{k<j}\left[-\ln \left(\cos \left(\theta_{j}-\theta_{k}\right)+1\right)+h\left(\rho_{j k}\right)\right]$,

where $\mathbf{x}_{j}=\mathrm{e}^{i \theta_{j}}$, and $\rho_{j k}=\left|\mathbf{r}_{j k}\right|$. The existence of a control law for which $V_{n}$ serves as a Lyapunov function (but not a global Lyapunov function) has been established [8]. However, proving convergence for control law (58) is the subject of on-going work.

Fig. 4 shows formation initialization for several sets of random initial conditions. Observe that the ultimate heading direction for the formation is initial-condition dependent. Also, observe that the relative positions of the vehicles within the formation is somewhat irregular, although the spacings between neighbors are roughly consistent. It appears from simulation results
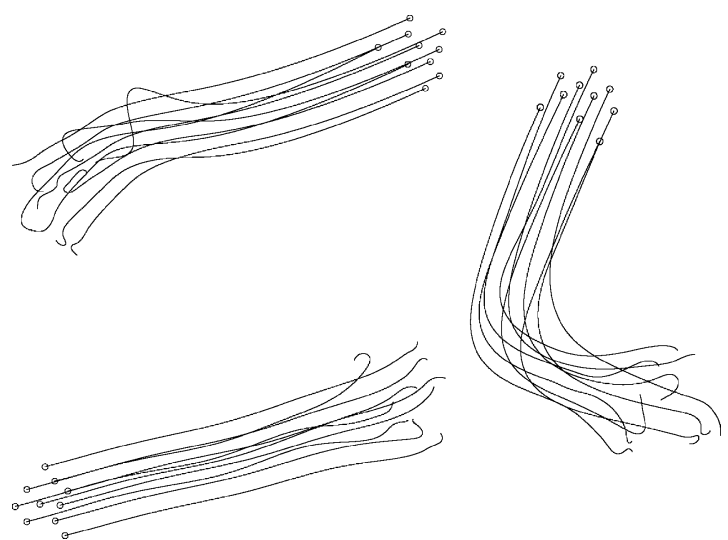

Fig. 4. Formation initialization for ten vehicles with three different sets of random initial conditions. In Eq. (58), $f(\cdot)$ is given by Eq. (7) and $\eta=\mu=\alpha=$ constant. 
that control law (58) does indeed avoid collisions and maintain swarm cohesiveness.

\subsection{Equilibrium formations}

The notions of configuration space and shape space described in Section 3 for the two-vehicle problem generalize naturally to $n$ vehicles in the plane. The configuration space consists of $n$ copies of $G=S E(2)$, and an appropriate non-collision manifold $M_{\text {config }}$ can be defined. The dynamics in configuration variables can be expressed as

$\dot{g}_{1}=g_{1} \xi_{1}, \quad \dot{g}_{2}=g_{2} \xi_{2}, \ldots, \quad \dot{g}_{n}=g_{n} \xi_{n}$,

where $\xi_{1}, \xi_{2}, \ldots, \xi_{n} \in \mathfrak{g}$ have the form

$\xi_{j}=A_{0}+A_{1} u_{j}, \quad j=1, \ldots, n$.

We define the shape variables

$\tilde{g}_{j}=g_{1}^{-1} g_{j}, \quad j=2, \ldots, n$,

which evolve on the reduced (shape) space consisting of a product of $n-1$ copies of $G$. (It is also possible to define shape variables in other combinations; e.g., $\tilde{g}_{j}=$ $g_{j-1}^{-1} g_{j}, j=2, \ldots, n[10]$.) Here we have assumed that $\xi_{1}, \xi_{2}, \ldots, \xi_{n}$ depend only on the reduced variables $\tilde{g}_{2}, \tilde{g}_{3}, \ldots, \tilde{g}_{n}$, and we note that this assumption can indeed be verified for control law (58).

By calculations analogous to those in Section 3.3, we are led to the conclusion that at equilibrium (in shape space), $u_{1}=u_{2}=\cdots=u_{n}$.

Proposition 4. Consider the dynamics given by Eqs. (60) and (61), evolving on the collision-free submanifold

$$
\begin{aligned}
M_{\text {config }}= & \{\left(g_{1}, g_{2}, \ldots, g_{n}\right) \in \overbrace{G \times G \times \cdots \times G}^{n \text { copies }} \mid \\
& \left.\mathbf{r}_{k} \neq \mathbf{r}_{j}, 1 \leqslant j \neq k \leqslant n\right\},
\end{aligned}
$$

where $G=S E(2)$ (and $\mathbf{r}_{j}$ is defined as in Eq. (21) for $j=1, \ldots, n)$. Assume that the controls $u_{1}, u_{2}, \ldots, u_{n}$ depend only on the shape variables given by Eq. (62) (i.e., the controls are G-invariant). Then for

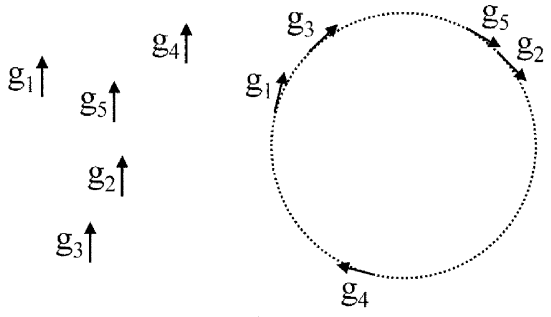

(a)

(b)

Fig. 5. Relative equilibria for the $n$-vehicle problem, illustrated for $n=5$ (arrows indicate tangent vectors to the vehicle trajectories): (a) for $u_{1}=u_{2}=\cdots=u_{n}=0$, and (b) for $u_{1}=u_{2}=\cdots=u_{n} \neq 0$.

equilibrium shapes (i.e., for relative equilibria of the dynamics (60) on configuration space) $u_{1}=u_{2}=\cdots=$ $u_{n}$, and there are only two possibilities:

(a) $u_{1}=u_{2}=\cdots=u_{n}=0$ at equilibrium, in which case a relative equilibrium consists of all vehicles heading in the same direction (with arbitrary relative positions within the formation), or

(b) $u_{1}=u_{2}=\cdots=u_{n} \neq 0$, in which case a relative equilibrium consists of all vehicles moving on the same circular orbit, with arbitrary chordal distances between them.

Proof. Omitted due to space constraints, but similar to the proof of Proposition 2 (see [7]).

Fig. 5 illustrates the two types of relative equilibria for the $n$-vehicle problem described in Proposition 4.

\subsection{Stabilizing control laws}

Thus, the control system (5), or system (57) and (58), can be naturally understood in the Lie group setting. This suggests that shape space notions for Lie groups may play an important role in formation control problems. We have shown how to characterize the set of all possible relative equilibria for any choice of $G$-invariant control law.

Based on simulation results, control law (58) appears to stabilize a relative equilibrium with $u_{1}=u_{2}=$ $\cdots=u_{n}=0$. A control law which appears in simulation to stabilize a relative equilibrium with $u_{1}=u_{2}=$ 


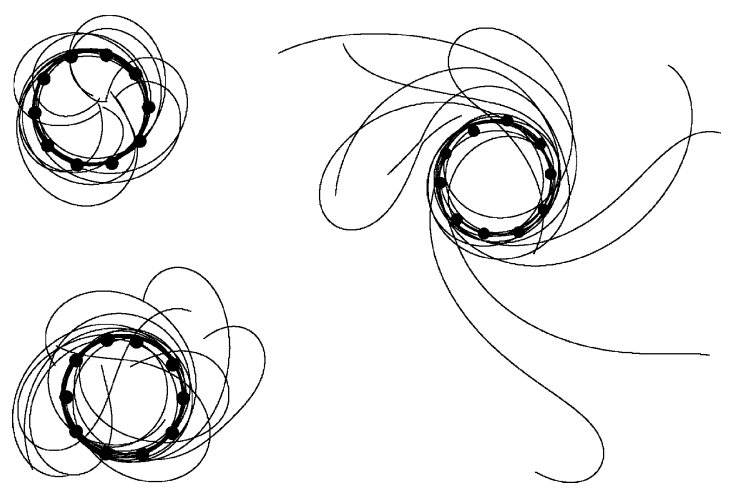

Fig. 6. Circular formation initialization for ten vehicles from three different sets of random initial conditions.

$\cdots=u_{n} \neq 0$ is

$u_{j}=\frac{1}{n} \sum_{k \neq j}\left[\eta\left(\frac{\mathbf{r}_{j k}}{\left|\mathbf{r}_{j k}\right|} \cdot \mathbf{x}_{j}\right)-f\left(\left|\mathbf{r}_{j k}\right|\right)\left(\frac{\mathbf{r}_{j k}}{\left|\mathbf{r}_{j k}\right|} \cdot \mathbf{y}_{j}\right)\right]$,

$j=1, \ldots, n$, where, as for Eq. (58), $\mathbf{r}_{j k}=\mathbf{r}_{j}-\mathbf{r}_{k}$, and $f(\cdot)$ may be given by equation (7); but here $\eta$ may be either positive or negative.

Fig. 6 shows simulation results for control law (64). Eq. (64), like Eq. (58) for the rectilinear control law, can be expressed in terms of shape variables alone.

There is a simple physical motivation for the circular control law (64), just as there was for the rectilinear control law (58). Each term involving $\eta$ tends to align vehicle $j$ with the baseline between vehicle $j$ and vehicle $k$, but in addition, vehicle $j$ tries to keep vehicle $k$ to its left or right, depending on the sign of $\eta$. As in the rectilinear case, the terms involving $f(\cdot)$ cause vehicle $j$ to steer toward or away from vehicle $k$ to maintain appropriate separation.

\subsection{Guiding formations}

The multi-vehicle control laws given by Eqs. (58) or (64) are starting points for the design of formation controllers for specific tasks. In particular, the $S E(2)$ symmetry that allows a reduction of the dynamics from configuration space to shape space means that the steady-state configuration-e.g., the heading direction for the rectilinear control law (58) or the center of
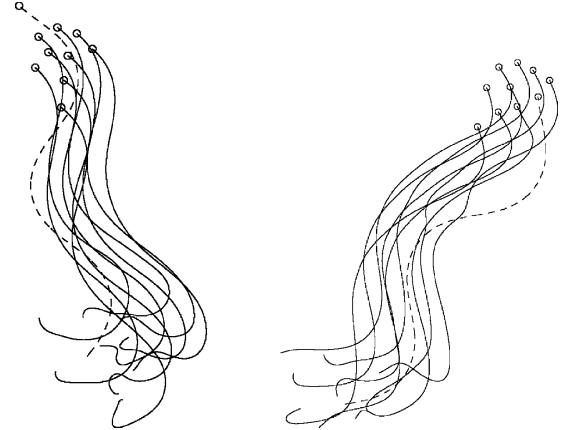

Fig. 7. Leader-following behavior: one vehicle (dashed line) follows a prescribed steering program unaffected by the other vehicles (for two different sets of random initial conditions).

the circular orbit for the circular control law (64) will be initial-condition-dependent. As mentioned in Section 4, in practical situations, it is often preferable to direct the formation toward some particular location, e.g., by introducing waypoints or beacons (which break the $S E(2)$ symmetry of the control law). Another possibility for the rectilinear control law (58) is leader-following behavior, which can be implemented simply by prescribing a steering program $u_{j_{0}}$ for vehicle $j_{0}$. The other vehicles obey the same interaction law as before, but vehicle $j_{0}$ ignores the other vehicles and instead follows its prescribed trajectory, as illustrated in Fig. 7.

Another technique for dynamically reconfiguring the formation is through on-the-fly parameter modification. For example, changing the length-scale parameter $r_{0}$ in the rectilinear control law (58) changes the steady-state intervehicle distance, and hence the extent of the formation (transverse to the direction of motion), as illustrated in Fig. 8. Introducing mollifiers, i.e., allowing $\eta, \alpha$, and $\mu$ to depend (in a monotonically decreasing way) on $\left|\mathbf{r}_{j k}\right|$ in Eq. (58) or (64), provides a natural mechanism for handling the interaction between distinct formations which come into proximity of each other. (For an alternative approach based on graph-theoretic methods, see [5], which considers a model proposed by Vicsek, et al. [18]. The Vicsek model is a discrete-time, unit-speed model in which each vehicle updates its heading direction at each time step by averaging its current heading direction with those of its neighbors located within a fixed 


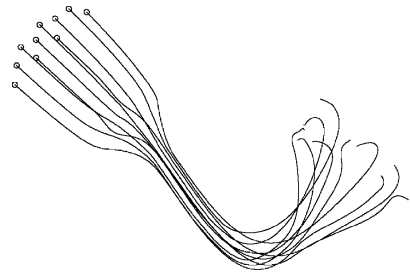

(a)

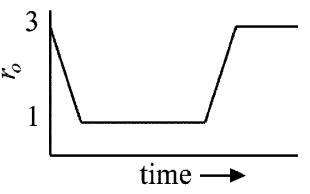

(b)

Fig. 8. On-the-fly modification of the separation parameter $r_{0}$ : (a) the vehicle trajectories; and (b) a semilog plot (with linear time axis) of the normalized separation parameter.

distance of itself. A noise term also contributes to the heading-direction updates.)

\subsection{Sensing and implementation issues}

A feature of the control laws (58) and (64) is that all of the quantities appearing in the expressions for the controls are simple functions of distance and angle measurements between pairs of vehicles. These quantities can, in principle, be obtained without reliance on GPS measurements. One technique, which uses pairs of antennas on each vehicle for radio-frequency measurement of the required distances and angles is outlined in [7]. Alternatively, optical sensing and/or acoustic sensing could be used, and there may be connections with sensing in biological swarming and schooling systems. Such a sensor-based implementation might be appropriate for large formations of simple vehicles.

Our original practical motivation was, however, to control small formations of UAVs equipped with GPS. In this context, the simplicity of the control laws (58) and (64) enables their real time implementation within the vehicles' limited on-board processing capability. Because of the high speeds of the vehicles, and the limited capacity (and imperfect reliability) of their wireless communications links, it may not be practical to exchange GPS information at each time step of (a time-discretized version of) the control algorithm. Therefore, an alternative approach in which each vehicle simulates the evolution of the entire formation, and communication is only used to correct for estimation errors, is reasonable in this context, and motion-description-language concepts are helpful [13].

\section{Future research directions}

Our work on formation control using models based on the Frenet-Serret equations of motion is still in its early stages. Areas of current and future work include developing control laws and proving stability results for formations of $n$ vehicles, investigating continuum limits in which the number of "vehicles" becomes infinite, and investigating three-dimensional formation-control problems. (A preliminary formulation of a continuum model appears in [8].) We are also studying obstacle avoidance, where our approach to formations based on gyroscopic forces leads to a natural approach to obstacle avoidance based on gyroscopic forces $[2,15,21]$.

\section{Acknowledgements}

The authors would like to thank Jeff Heyer, Larry Schuette, Jason Fox, Ted Roberts, and Hal Levitt of the Naval Research Laboratory, Washington, DC for valuable discussions.

\section{References}

[1] W.M. Boothby, An Introduction to Differentiable Manifolds and Riemannian Geometry, 2nd Edition, Academic Press, Orlando, 1986.

[2] D.E. Chang, S. Shadden, J.E. Marsden, R. Olfati-Saber, Collision avoidance for multiple agent systems, IEEE Conference on Decision and Control, 2003, in press.

[3] A. Czirók, M. Matsushita, T. Vicsek, Theory of periodic swarming of bacteria: application to Proteus mirabilis, Phys. Rev. E 63 (3) (2001), 031915-1-031915-11.

[4] D. Grünbaum, Schooling as a strategy for taxis in a noisy environment, in: J.K. Parrish, W.M. Hamner (Eds.), Animal Groups in Three Dimensions, Cambridge University Press, Cambridge, 1997.

[5] A. Jadbabaie, J. Lin, A.S. Morse, Coordination of groups of mobile autonomous agents using nearest neighbor rules, IEEE Trans. Automat. Control 48 (6) (2003) 988-1001 (also in Proceedings of the IEEE Conference on Decision and Control, Vol. 3, 2002, pp. 2953-2958).

[6] V. Jurdjevic, Geometric Control Theory, Cambridge University Press, Cambridge, 1997.

[7] E.W. Justh, P.S. Krishnaprasad, A simple control law for UAV formation flying, Institute for Systems Research Technical Report TR 2002-38, 2002. 
[8] E.W. Justh, P.S. Krishnaprasad, Steering laws and continuum models for planar formations, Proceedings of the IEEE Conference on Decision and Control, 2003, in press.

[9] H. Khalil, Nonlinear Systems, Macmillan, New York, 1992.

[10] P.S. Krishnaprasad, D.P. Tsakiris, G-snakes: nonholonomic kinematic chains on Lie groups, Proceedings of the IEEE Conference on Decision and Control, Vol. 3, 1994, pp. 2955 -2960 (see also Institute for Systems Research Technical Report ISR TR 94-27, 1994).

[11] H. Levine, W.-J. Rappel, I. Cohen, Self-organization in systems of self-propelled particles, Phys. Rev. E 63 (1) (2000) 017101-1-017101-4.

[12] R.G. Littlejohn, M. Reinsch, Gauge fields in the separation of rotations and internal motions in the $n$-body problem, Rev. Modern Phys. 69 (1) (1997) 213-275.

[13] V. Manikonda, P.S. Krishnaprasad, J. Hendler, Languages, behaviors, hybrid architectures, and motion control, in: J. Baillieul, J.C. Willems (Eds.), Mathematical Control Theory, Springer, New York, 1999, pp. 199-226.

[14] P. Ogren, E. Fiorelli, N.E. Leonard, Formations with a mission: stable coordination of vehicle group maneuvers, Proceedings of the MTNS, 2002.
[15] L. Singh, H. Stephanou, J. Wen, Real-time robot motion control with circulatory fields, Proceedings of the IEEE International Conference on Robotics and Automation, Vol. 3, 1996, pp. 2737-2742.

[16] T.R. Smith, H. Hanssmann, N.E. Leonard, Orientation control of multiple underwater vehicles with symmetry-breaking potentials, Proceedings of the IEEE Conference on Decision and Control, Vol. 5, 2001, 4598-4603.

[17] J. Toner, Y. Tu, Flocks, herds, and schools: a quantitative theory of flocking, Phys. Rev. E 58 (4) (1998) 4828 4858.

[18] T. Vicsek, A. Czirok, E.B.-Jacob, I. Cohen, O. Shochet, Novel type of phase transitions in a system of self-driven particles, Phys. Rev. Lett. 75 (1995) 1226-1229.

[19] L.-S. Wang, P.S. Krishnaprasad, Gyroscopic control and stabilization, J. Nonlinear Sci. 2 (1992) 367-415.

[20] F. Zhang, M. Goldgeier, P.S. Krishnaprasad, Control of small formations using shape coordinates, Proceedings of the IEEE International Conference on Robotics and Automation, 2003, pp. $2510-2515$.

[21] F. Zhang, E. Justh, P.S. Krishnaprasad, Guidance and obstacle avoidance algorithms for UAVs, Preprint, 2003. 